\title{
RECONFIGURACIÓN DE LA ECONOMÍA REGIONAL EN MÉXICO 1970-1995
}

\section{RESUMEN}

El trabajo tiene como objetivo explorar los cambios sectoriales y regionales que experimentó la economía mexicana durante el periodo 1970-1995. Esto es posibleal revisar brevemente los grandes cambios estructural es dela economía mexicana que caracterizaron este periodo y la dinámica de las regiones que presentan las 32 entidades federativas del país en sus aspectos sectorial y regional. En la primera parte se estudia el comportamiento del Producto Interno Bruto (PIB) de todos los 32 estados que integran el país, y está compuesta por nueve grandes divisiones de la actividad económica. Para el análisis de esta información se aplicó un análisis multivariado de componentes principales, cuyos resultados se procesaron posteriormente con un algoritmo de clasificación de punto variable. La clasificación obtenida y su expresión en mapas para los años de 1970, 1980, 1985 y 1995 hicieron posible identificar las tendencias territoriales del desarrollo sectorial de la economía mexicana durante el periodo estudiado.

Palabras clave: Economía, Desarrollo regional, Desarrollo sectorial, Cambios estructurales, PIB.

\section{ABSTRACT}

The work has as an objective to explore the sectorial and regional changes that experienced the Mexican economy during the period of 1970-1995. This has been made making an overview of the structural changes of the Mexican economy, which has been characteristic of this period, also studying the dynamics of the regions represented by the 32 states of the country in their sectorial and regional aspects. In the first hand it was studied the behavior of the Gross Domestic Product (GDP) of the 32 states which integrate Mexico in nine big divisions of the economic activity. For the analysis of this information it was applied a multivariateanalysis of principal components whoseresults were processed later

\footnotetext{
* DiRECTOR E INVESTIGADOR DEL INSTITUTO DE INVESTIGACIONES SOCIALES DE LA UNIVERSIDAD AUTÓNOMA DE BAJA CALIFORNIA. Correo electrónico: aranfla@uabc.mx
}

Artículo recibido en junio de 2003.

Artículo aprobado en octubre de 2003. 
Estudios Fronterizos, Nueva época, vol. 4, núm. 7, enero-junio de 2003

with an algorithm of classification of variable point. The obtained classification and their expression in maps for the years of 1970, 1980, 1985 and 1995 made possible to identify the territorial tendencies of the sectorial development of the Mexican economy during the period studied.

Keywords: Economy, Regional development, Sectorial development, Structural changes, GDP.

\section{INTRODUCCIÓN ${ }^{1}$}

\section{ANTECEDENTES}

En medio degrandes desequilibrios, en diciembre de $1982 \mathrm{Miguel}$ dela Madrid Hurtado asumió la presidencia de México y anunció un plan de emergencia que tenía como objetivos restablecer, en el corto plazo, la estabilidad financiera y controlar la inflación. En el mediano plazo proponía como metas fomentar la competitividad externa dela economía, fomentar el ahorro interno y disminuir la intervención estatal en la actividad económica.

La primera etapa del programa anunciado en el mes de diciembre se desarrolló de 1983 a 1985, dando un énfasis particular en la estabilización de la economía por medio de la corrección de los desequilibrios presupuestales y dela bal anza de pagos, y buscando, paralelamente disminuir la inflación (Lustig, 1994:146-153). Una segunda etapa sellevó a cabo de mediados de 1985 a diciembre de 1987, y buscó una mayor apertura comercial, para lo quese eliminaron los controles cuantitativos, lo que permitió en diciembre de 1987 la producción interna protegida por precios oficiales fuera prácticamente nula. La tercera etapa, estaba rel acionada con la introducción del pacto de solidaridad económica del periodo de 1989-1991, que logró profundizar la reforma estructural por medio de los procesos de privatización de los bancos, y más tarde el acuerdo de libre comercio con los Estados Unidos y Canadá,

${ }^{1}$ Documento presentado en la Reunión de Especialistas sobre Desarrollo Regional y Urbano En M éxico: Interpretaciones, Experiencias y Nuevas Tecnologías, efectuada del 26 al 27 octubre de 2000 en El Colegio de Ingenieros Civiles de México, A.C., en México, D.F. 
quesefirmó en noviembre de 1993y entró en vigor el 1 deenero de 1994.

A lo largo de este proceso es posible identificar una preocupación continua por un ambienteinterno y externo caracterizado por la incertidumbre económica y financiera, así como una presencia creciente de reformas estructurales que marcarían el futuro de la ruta emprendida por la economía mexicana.

Esta decisión y el proceso originado -y que aún, hoy día, no termina de completarse - dejan ver tres medidas quemarcaron la nueva fase en la que entró la economía mexicana: la primera fue el ingreso de México al Acuerdo General de A ranceles y Comercio (GATT) en 1986, que oficializó la liberalización comercial del país y se convirtió en la condición impuesta por acreedores y organismos internacionales para continuar apoyando al gobierno mexicano en el manejo de la crisis financiera; la segunda fueron las reformas a la ley de inversión extranjera y de diversos sectores, para abandonar la utilización de criterios discriminatorios y promover la creación de reglas de juego generales, neutrales y estables para facilitar la libre operación de los agentes económicos; y una tercera medida, fue la transferencia deempresas públicasal sector privado y la instrumentación deesquemas deconcesión para promover una mayor eficiencia del sector productivo en servicios queanteriormente prestaba el gobierno. En estas medidas destaca una reforma de la naturaleza del Estado y de sus funciones, un reconocimiento al rel evo en la esfera económica del capital privado y la creación acelerada de un nuevo marco normativo en las relaciones económicas de México hacia fuera y hacia adentro (Lora y Barrera , 1998:55-60).

\section{LOS CAMBIOSY EL AMBIENTE DE NEGOCIOS}

El recuento anterior permite distinguir una relación dependiente del sector externo en la economía mexicana a lo largo del periodo estudiado y un mejor posicionamiento a finales del mismo en la economía mundial, resultado del proceso de apertura que 
Estudios Fronterizos, Nueva época, vol. 4, núm. 7, enero-junio de 2003

emprendió el gobierno mexicano y su modo de inserción en la economía mundial.

En 1984 la economía mexicana se ubicaba en el número 28 entre los principales países exportadores del mundo, y para 1995 ocupaba el lugar 16 (Cervantes, 1996:179); si se toma en cuenta que los intercambios mundiales crecieron, el cambio registrado por México resulta doblemente significativo.

Este proceso marca una nueva etapa en la economía del país, guiada por la racionalidad del capital en el ambiente general de los negocios, en dondetuvo lugar un crecimiento sostenido de las exportaciones no petroleras, que de constituir $44.8 \%$ del total de las exportaciones en 1985, diez años después aumentó a 89.4\%. El cambio que resulta significativo fue producto del crecimiento de la industria manufacturera, que de representar $37.6 \%$ del total de las exportaciones en 1985, para 1995 había crecido en una proporción 83.7\% (Cervantes, 1996:183). Estos resultados indican que se cumplió el propósito de despetrolizar al sector externo de la economía, aunque permanecela incógnita sobrelos encadenamientos productivos que estas exportaciones generan sectorial y regionalmente.

Durante el periodo de 1980 a 1995, como resultado del entorno económico volátil, se produjeron altibajos en la producción en general y se mantuvo un crecimiento continuo de la producción industrial y en particular la manufacturera. En este sector destaca la emergencia degrandes conglomerados mexicanos queduranteeste periodo consolidaron posiciones importantes en el mercado mundial deproductos, como son los casos de CEMEX, BIMBO, HYLSA, VISA y Cervecería Modelo, empresas que expandieron sus mercados en el exterior y que abrieron plantas principalmente en las mayores zonas metropolitanas del país y las ciudades medias como estrategia para hacer frente a la volatilidad macroeconómica durante este periodo (Rendón, 1997: 53-70). También en este sector se concentró la producción de la industria maquiladora de exportación la cual en sus primeras etapas experimentó un acel erado y continuo crecimiento en la región fronteriza con los Estados Unidos, y que después de 1982 se desplegó también hacia las mayores zonas 
metropolitanas y a ciudades localizadas en diferentes regiones del país.

La característica del ambiente de negocios durante este periodo tenía que ver con el cambio de la dirección económica que cedió el Estado al capital privado. Como parte de la lógica de la racionalidad económica y regional del Estado, la política de fomento a la industria y a las actividades productivas tenía el propósito dedetonar regiones en el marco dereducir los desequilibrios territoriales, mientras queen la lógica del capital sebusca aprovechar las diferencias de local ización, dotación derecursos y combinación deprocesos, independientementedelos desequilibrios que los propósitos de cada una de las corporaciones nacionales o extranjeras pudieran traer. Dicho de otra manera, en una lógica corporativa que cada vez tiende a ser más plurisectorial y plurirregional , los resultados locales tienen mayor importancia y prioridad que la noción de equilibrio regional desde una perspectiva nacional.

En estas condiciones, las comunidades locales y los gobiernos estatales han venido promoviendo sus territorios a la demanda de inversionistas nacional es y extranjeros, ofertando toda una serie de ventajas competitivas quetienen como propósito establecer las diferencias (ITESM, 1999:13-19) y entrar a un mercado en donde las ventajas se definen en razón de los intereses generales de las corporaciones y en respuesta a las ventajas comparativas que ofrecen las regiones, por la capacidad quetienen estas comunidades económicas y de la sociedad para atraer nuevos capitales e inversiones productivas que garanticen su crecimiento. En esta dirección varias interpretaciones coinciden en señal ar esta capacidad como determinante en el desarrollo regional sobre la base dela cooperación y su atracción como resultante, la competitividad que pueda lograr la región (Azúa, 2000: 69-84), o bien la competitividad como producto de las estructuras y condiciones regionales quela llevan a adaptarsea un entorno cambiante (Ruiz Durán, 1999:13-56) reconociendo con ello el papel activo que adquieren las regiones y queresponden a sus recursos, instituciones públicas, empresariales y dela sociedad. 
Estudios Fronterizos, Nueva época, vol. 4, núm. 7, enero-junio de 2003

DINÁMICA Y COMPOSICIÓN DE LA PRODUCCIÓN REGIONAL

Las condiciones macroeconómicas son determinantes en la orientación sectorial y regional de la producción. Las grandes tendencias entre las que se encuentran los cambios institucional es como los que experimentó el país durante el periodo estudiado fueron determinantes en el ajuste y desempeño de las economías regionales. Asimismo fue determinante porque la apertura económica afectó de manera directa los principios básicos de la política industrial que se había seguido durante varias décadas de manera que la apertura significó cambios sustantivos en la política industrial que, de la promoción, se desplazó hacia una liberación que dejó su desarrollo al juego del mercado.

Esteapartado tiene el propósito de evaluar los cambios en tres aspectos: la dinámica y los ciclos en la economía nacional; el tránsito sectorial registrado por medio del PIB en el país; y la dinámica deespecialización y diversificación en las diferentes economías regionales del país.

\section{DINÁMICA DEL PIB}

A nalizar la evolución dela producción en el país duranteel periodo mencionado (1970-1995), tiene como principal propósito establecer un marco general dela evolución dela producción para un periodo reconocido como crítico en el desarrollo contemporáneo de México. Durante este periodo se presentaron tres etapas: el periodo de expansión caracterizado por un fuerte gasto gubernamental financiado con impresión monetaria y endeudamiento externo; la crisis dela deuda que tuvo su momento crítico en 1982; y la estabilización, el cambio estructural y el colapso quetuvo lugar en diciembre de 1994 (Cárdenas, 1996:80-180).

Estas etapas relevantes en la historia reciente de la economía mexicana estuvieron caracterizadas por cambios de natural eza más compleja como son: la apertura de la economía mexicana y la reforma del Estado que se llevó a cabo durante todos estos años y que no estaba desligada de los procesos económicos descritos; el 
desprendimiento gradual delos poderes regionalesquesesucedieron durante el periodo y que comenzaron a dar indicios de una competencia regional entre las diferentes entidades federativas de las inversiones vis a vis y una redistribución de las actividades productivas en el territorio nacional que confirma la tendencia hacia la urbanización de la sociedad mexicana que se manifiesta en el despliegue de las actividades productivas hacia las principales zonas metropolitanas del país y cada vez con mayor frecuencia hacia las ciudades medias que han experimentado un rápido crecimiento en las últimas décadas (Aguilar y Graizbord, 1996:31-48).

En México, el proceso de ajuste estructural se desenvolvió en el marco deuna gran incertidumbrey volatilidad macroeconómica derivada en la primera parte del período de las políticas internas en las principal es variables monetarias, en donde los activos petroleros respaldaron los compromisos financieros de M éxico con el exterior. Mientras en la segunda parte, los efectos impulsados desde el exterior combinaron el aumento en las tasas de interés y el deterioro de los precios del petróleo como principales factores de la inestabilidad macroeconómica en el interior del país, al mismo tiempo que se instrumentaban medidas de renegociación de la deuda pública.

Todos estos factores enrarecieron el entorno económico del país y seagregaron a los cambios estructurales que gradual menteel gobierno mexicano fue instrumentando y quemotivaron una serie de cambios y relevos en la base productiva y los patrones de organización dentro de la misma. Esta dinámica se reflejó en varios niveles: el gobierno mexicano se desprendió a lo largo de este periodo de la mayor parte de sus activos incorporados en la producción; el sector productivo nacional experimentó una transformación de donde derivó el cierre de un número importante de plantas y la expansión de un número reducido de corporativos, que poco a poco se extendieron en el territorio nacional y trascendieron con inversiones en otros países; estas industrias se concentraron de manera predominante en el sector alimentos y bebidas, cemento y servicios. Por otra parte, la industria 
maquiladora de exportación creció de una manera acelerada superando a lo largo del periodo a la mayor parte de las actividades económicas del país, en particular las industrias automotriz y electrónica que se concentraron en este tipo de plantas.

FIGURA 1. México, dinámica del PIB en 1971-1994.

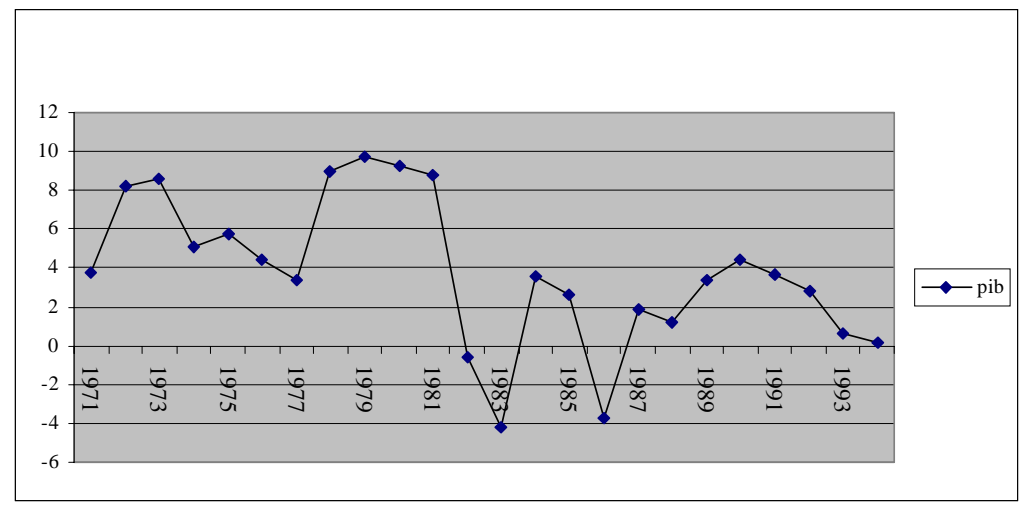

FUENTE: Producto interno bruto por entidad federativa, INEGI, 1999.

Durante todo este periodo, la dinámica del PIB (ver figura 1) mostró dos etapas en su trayectoria: la primera de ellas, una dinámica positiva de 1971 a 1982, tiempo en el cual sepresentaron una serie de bajas entre los años 1974 y 1977, debi do a una situación de incertidumbrequeculminó con la devaluación de 1976, dio inicio a una fase de mucha volatilidad en la economía mexicana. En la segunda etapa 1982-1994 se identifica la mayor inflexión en el crecimiento del PIB la cual alcanza su nivel más bajo en 1983, como consecuencia de la crisis de la deuda con una recaída del PIB el periodo 1985-1987 causada por el desplome de los precios del petróleo y la incertidumbrepara hacer frenteal servicio dela deuda.

\section{DINÁMICA SECTORIAL}

La dinámica sectorial del PIB durante el periodo estudiado tiene dos características principales, la primera de el las es un cambio en su composición (ver figura 2). En 1970, las actividades 
agropecuarias mantenían una participación del $12.0 \%$, mientras que las manufacturas, así como el comercio y los servicios de restauración, participaron con poco más del $20 \%$ cada una deellas en el total del producto. Las actividades de servicios comunales y personales tenían una participación de $14 \%$. Estos resultados muestran que se trataba de una estructura de transición en donde coexistían las actividades primarias con actividades de otros productos - como son las manufacturas y el comercio y los servicios de restauración-, que indican una naturaleza preferentemente urbana en la especial ización regional.

FIgURA 2. México, dinámica sectorial del PIB en 1970-1995.

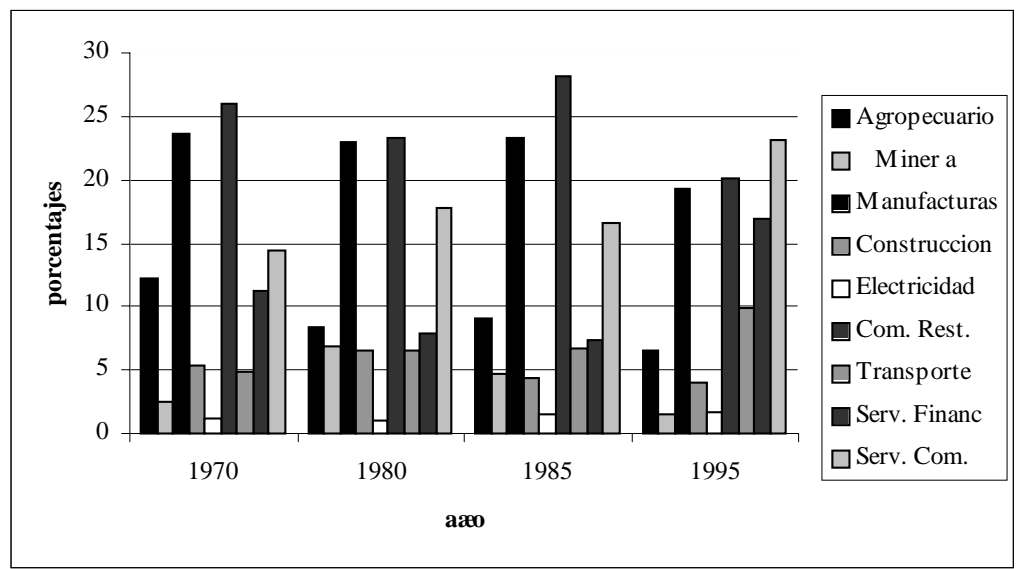

FuentE: Producto interno bruto por entidad federativa, INEGI, 1999.

La segunda característica ligada a la dinámica sectorial de este periodo, es que para 1995 la composición se había modificado y la participación de los sectores se había redistribuido. Esta estructura diversi ficada mostró una tendencia a equiparar la contribución delos sectores: la producción manufacturera se mantuvo con una participación cercana al 20\%; el comercio y los servicios de restauración también se redujeron, ubicándose alrededor del $20 \%$; mientras que los sectores de transporte, servicios financieros y 
Estudios Fronterizos, Nueva época, vol. 4, núm. 7, enero-junio de 2003

servicios comunal es aumentaron su participación para contribuir en conjunto con el 50\% del total del producto en 1995. De esta manera se puedeapreciar una diversificación en la composisición de la producción así como la consolidación de una producción de carácter urbano como consecuencia del descenso continuo de la participación del sector agropecuario, que en ese año contribuyó con $6 \%$ del total. Si bien la base productiva se diversificó durante el periodo, también se confirmó el predominio delas actividades no agrícolas en el país.

\section{ESPECIALIZACIÓN Y LA DIVERSIFICACIÓN REGIONAL}

El estudio de la dinámica de las estructuras productivas regionales tiene el propósito de analizar los cambios en la composición del producto interno bruto en los diez sectores que defineel Instituto N acional deEstadística Geografía e Informática (INEGI) ${ }^{2}$ en cada una de las entidades federativas del país. Gracias a la clasificación territorial y sectorial de estos conceptos, y utilizando un procedimiento estadístico multivariable, es posible identificar la reorganización en las actividades económicas y evaluar el reposicionamiento económico de cada una de las entidades federativas. Este procedimiento permite identificar algunos de los cambios estructurales experimentados a lo largo del periodo de 25 años para diez sectores productivos. También de esteanálisis estadístico permiteobtener resultados territoriales en los que se destaca el relevo de las posiciones y jerarquías de cada una de las entidades federativas, lo que facilita identificar patrones

2 INEGI, Clasificación mexicana de actividades y productos (CMAP), Censos económicos de 1994. Al seguir la clasificación de las actividades que contribuyen en la generación del producto interno bruto, son: 1) agricultura, ganadería, caza y pesca; 2) minería y extracción de petróleo; 3) industrias manufactureras (incluyen los establecimientos maquiladores); 4) electricidad y agua; 5) construcción; 6) comercio; 7) transportes y comunicaciones; 8) servicios financieros, de administración y alquiler de bienes muebles e inmuebles; 9) servicios comunales y sociales, profesionales y personales y; agregamos una variable: 10) servicios financieros imputados (que es una estimación del INEGI). 
geográficos y sus cambios a lo largo del periodo estudiado (ver figuras 3 y 4).

FIGURA 3. Dinámica de la diversificación regional del PIB por grandes divisiones económicas en México.

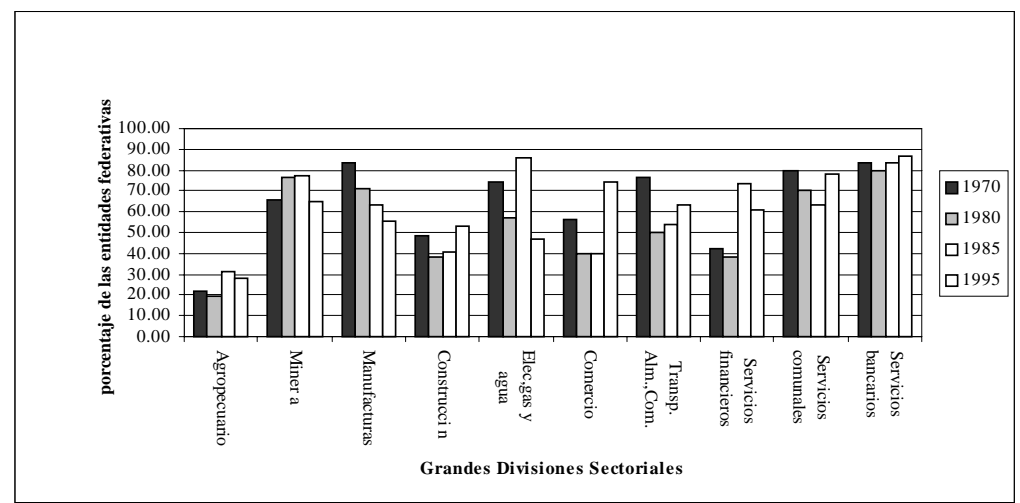

Fuente: Elaboración propia con base en Producto Interno Bruto, INEGI 1999.

FIGURA 4. Dinámica de la especialización regional del PIB por grandes divisiones económicas en México.

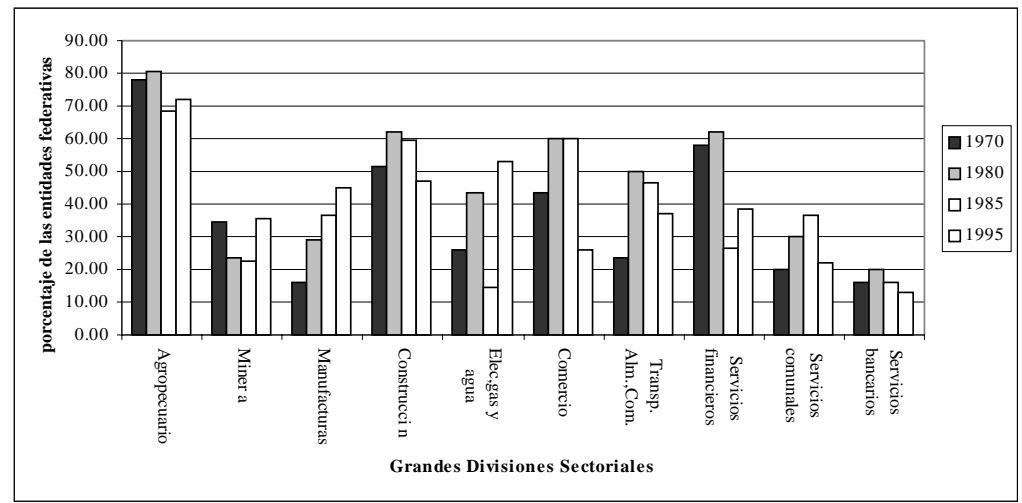

FUENTE: Elaboración propia con base en Producto Interno Bruto, INEGI 1999. 
A lo largo delos 25 años del periodo estudiado fueposibleidentificar cambios en la composición del PIB por cada uno delos diez sectores de origen. Dichos cambios fueron identificados con el apoyo de los perfiles sectorial es para cada uno de los cuatro años (1970, 1980, 1985 y 1995) empleados como referencia y, que se obtuvieron al aplicar de manera complementaria el método de componentes principales y un sistema de clasificación de punto móvil, que constituyen una metodología de análisis estadístico exploratorio multivariable (Lebart et al., 1995:7-10).

La primera evidencia encontrada es que hay tres perfiles de composición sectorial del producto: la primera correspondea 1970 y 1980, en donde se destaca la importancia que tienen los sectores de servicios comunales, sociales y profesionales; construcción; comercio, restaurantes y hoteles; transportes, al macenamiento y comunicaciones; servicios financieros, seguros y bienes inmuebles (figuras 5 y 6). Estas asociaciones destacan una estructura del PIB regional a una escal a nacional, en donde sobresalen las actividades de intercambio y los servicios ligados a la expansión de las actividades urbanas quecaracterizan esteperiodo, durante el cual continuó en aumento la población en las grandes zonas metropolitanas y en un número importante delas ciudades medias que se desarrollaron a lo largo y ancho del país (Aguilar y Graizbord, 1996:31-35).

La segunda etapa corresponde a 1985, en la que destaca la importancia quetienela partici pación de servicios financieros, seguros y bienes inmuebles y los servicios bancarios imputados en asociación con todos los otros sectores, como son: la construcción; servicios comunales, sociales y personales; transporte, almacenamiento y comunicaciones. Para este año es evidente la presencia del sector financiero en actividades predominantemente ligadas al intercambio, y reflejan la crisis económica y financiera por la que atravesaba el país durante ese tiempo (ver figura 7).

La tercera etapa que identificada corresponde a 1995, y en la quees posi ble identificar una economía sin asociaciones significativas y sin sectores dominantes. La estructura sectorial del PIB nacional confirma en sus resultados los efectos producto de las 
FIGURA 5. Tipología regional de la estructura sectorial del PIB en México 1970.

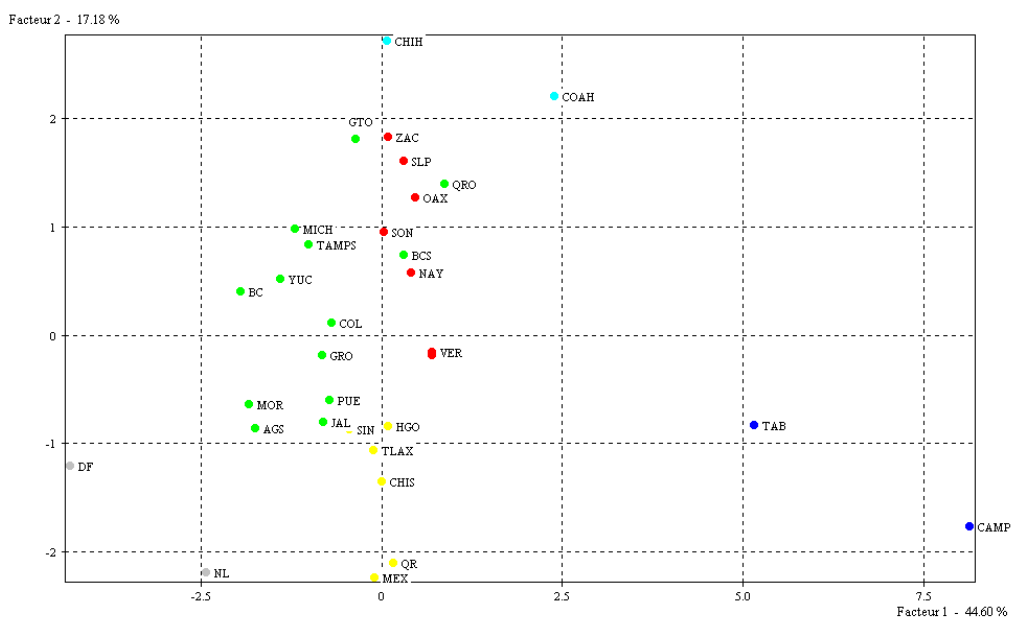

Fuente: Elaboración propia con base en Producto Interno Bruto, INEGI 1999.

FIGURA 6. Tipología regional de la estructura sectorial del PIB en M éxico 1980.

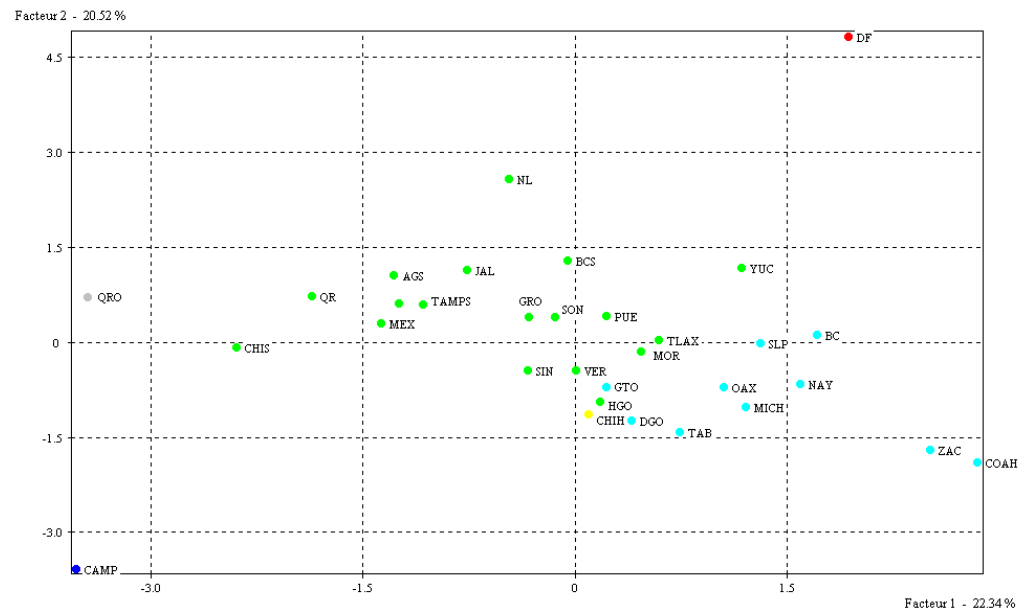

Fuente: Elaboración propia con base en Producto Interno Bruto, INEGI 1999. 
FIgURA 7. Tipología regional de la estructura sectorial del PIB en México 1985.

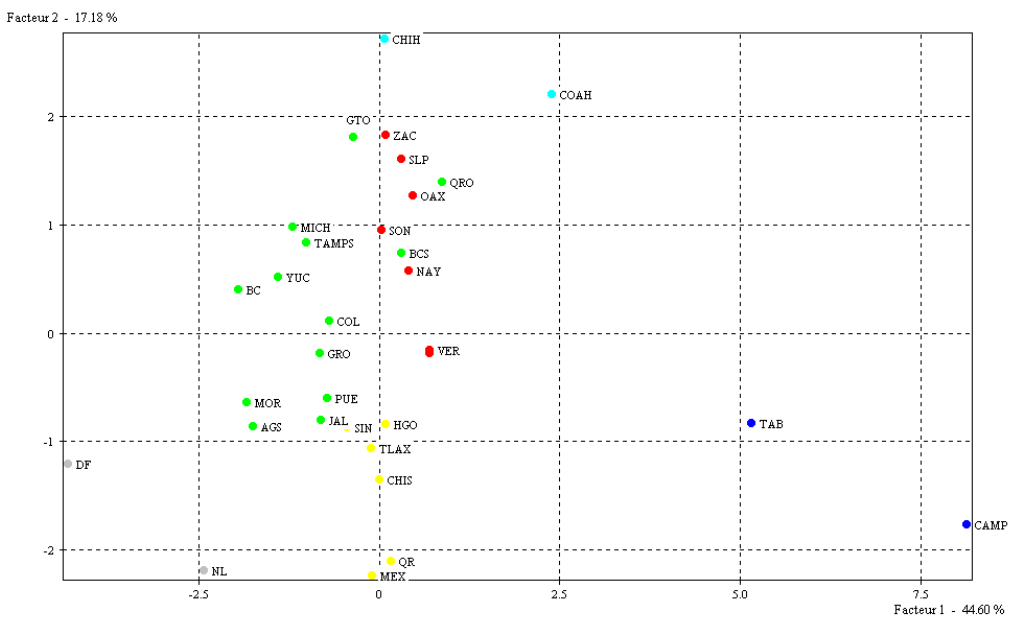

FUENTE: Elaboración propia con base en Producto Interno Bruto, INEGI 1999.

reformas estructural es aplicadas en los años precedentes y la desestabilización dela crisis de diciembreen 1994, cuyos resultados fueron: Ia ausencia de valores significativos en la matriz de correlaciones; las pocas asociaciones relevantes entre sectores; y un cambio drástico en los resultados registrados durante los años precedentes (ver figura 8).

LA DINÁMICA REGIONAL

UNA TIPOLOGÍA REGIONAL

Los cambios registrados en la estructura de la producción durante los últimos años, requieren profundizar en la identificación de las tendencias de especialización y de diversificación que derivan del indicador utilizado para este estudio, quevienen a ser resultados registrados en cada uno de los ejes factoriales. También fue 
FIGURA 8. Tipología regional de la estructura sectorial del PIB en México 1995.

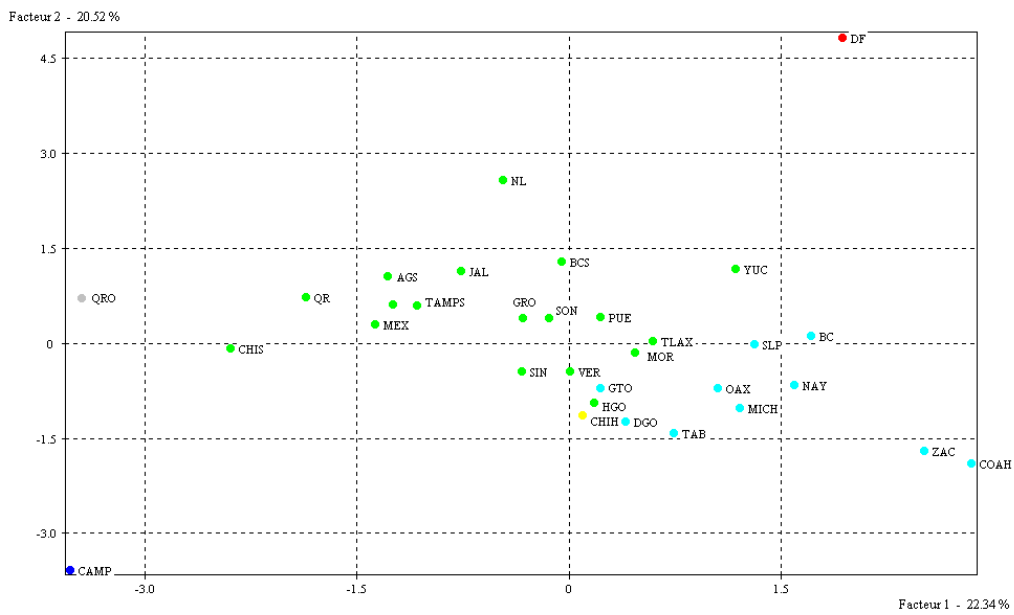

FueNTE: Elaboración propia con base en Producto Interno Bruto, INEGI 1999.

posible identificar los cambios experimentados a lo largo de este periodo; así la reestructuración económica como una gran tendencia decarácter macroeconómico, adquiere una dimensión diferente que es menester identificar y describir como parte de la dinámica de organización y operación del territorio durantelas casi tres décadas que integran este análisis.

Las variables originales de dicho análisis son los cocientes de especialización sectorial en el PIB regional, representados por las 32 entidades federativas. Estas variables procesadas y clasificadas por los ACP arrojaron resultados que se pueden describir en dos direcciones: la primera es una clasificación de las entidades federativas en cinco grupos, los cuales tienen como característica principal una homogeneidad. Estos agrupamientos serealizan para cada uno de los cortes transversales (1970, 1980, 1985 y 1995) que permiten identificar cambios del total y reacomodos entre los grupos. El factor de control en el análisis es únicamente el número de agrupamientos, y los resultados semuestran en las figuras 9 a la 12. 
FIGURA 9. Clasificación regional de la estructura de especialización sectorial del PIB por entidad federativa en México 1970.

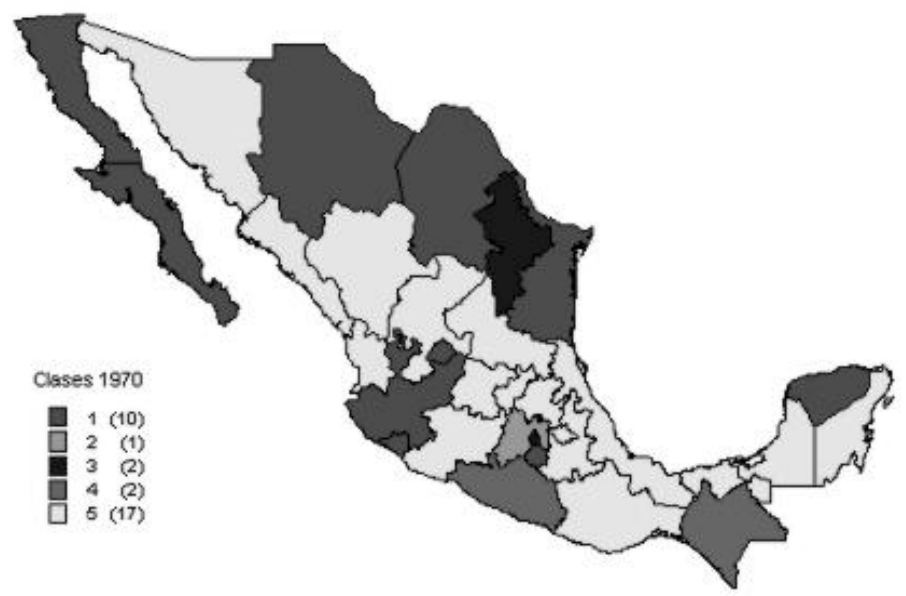

FUENTE: Elaboración propia con base en Producto Interno Bruto, INEGI 1999.

FIGURA 10: Clasificación regional de la estructura de especialización sectorial del PIB por entidad federativa en México 1980.

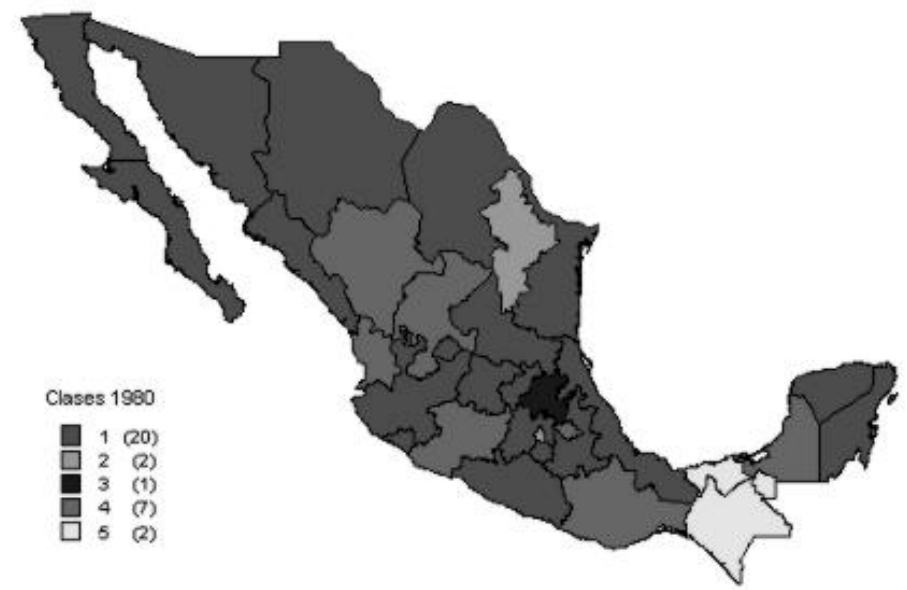

FUENTE: Elaboración propia con base en Producto Interno Bruto, INEGI 1999. 
FIGURA 11: Clasificación regional de la estructura de especialización sectorial del PIB por entidad federativa en México 1985.

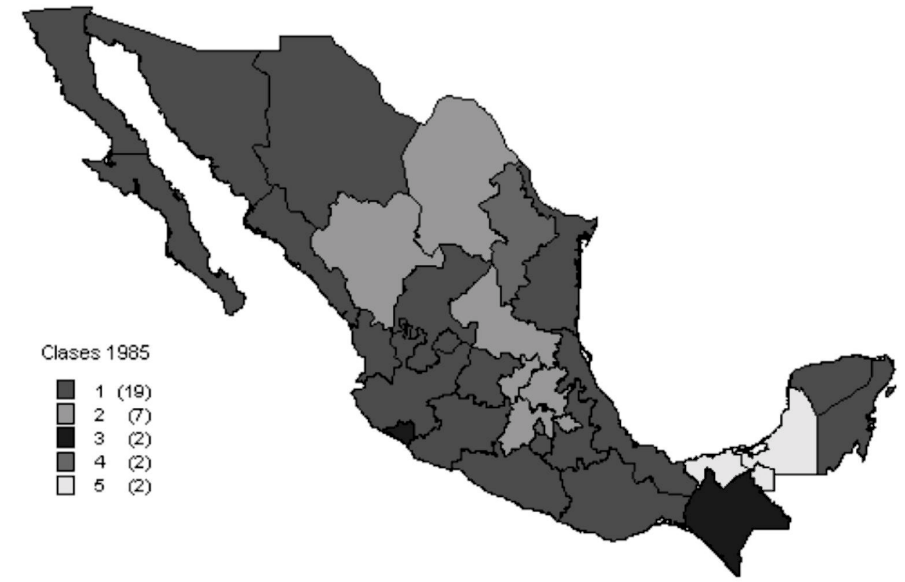

Fuente: Elaboración propia con base en Producto Interno Bruto, INEGI 1999.

FIGURA 12: Clasificación regional de la estructura de especialización sectorial del PIB por entidad federativa en México 1995.

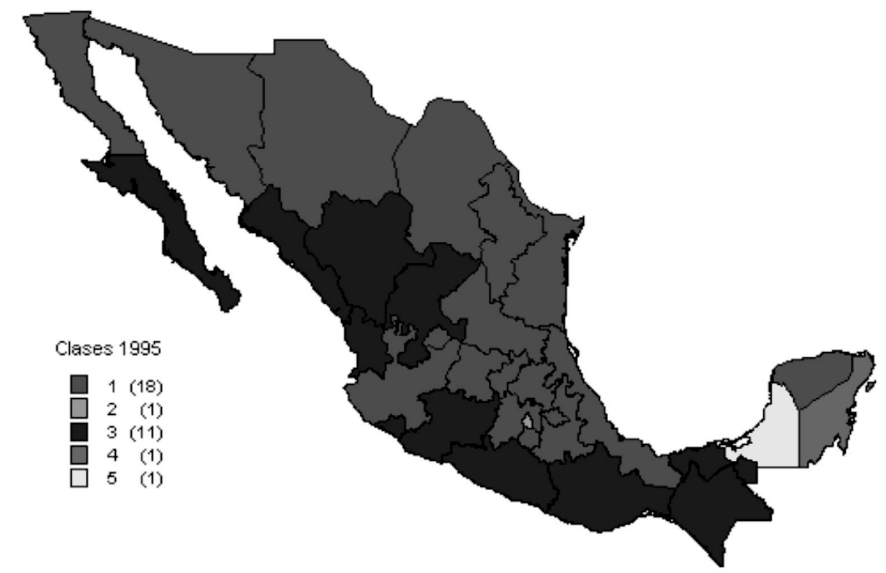

FuENTE: Elaboración propia con base en Producto Interno Bruto, INEGI 1999. 
Los resultados de los cambios registrados dan lugar a una redistribución de los agrupamientos en cada uno de los años y expresan el nivel de desigual dades existentes. En 1970, los agrupami entos mostraron por un lado, a la clase 1, integrada por las siguientes entidades: Aguascalientes, Baja California, Baja California Sur, Coahuila, Chihuahua, Jalisco, Morelos, Tamaulipas y Yucatán (ver figura 9). Por otra parte, está la clase 5, integrada por: Campeche, Colima, Durango, Guanajuato, Hidalgo, Michoacán, N ayarit, Oaxaca, Puebla, Querétaro, Quintana Roo, San Luis Potosí, Sinaloa, Sonora, Tabasco, Tlaxcala, Veracruz y Zacatecas. Y estas dos clases se encuentran el Estado de M éxico, Distrito Federal, Nuevo León, Chiapas y Guerrero, distribuidos entre las clases 2, 3 y 4.

En el año de 1970 el desarrollo regional mostraba marcados contrastes entre la clase 1 y la clase 5; la primera de ellas tuvo como sectores característicos el comercio, restaurantes y hoteles, y transportes, almacenamiento y comunicaciones, que puede decirsese trataba de actividades ligadas al turismo y al movimiento de mercancías y de personas y que fueron representativas de Aguascalientes -en el centro del país- y a la mayoría de las entidades fronterizas del norte. La clase 5, por su parte, se caracterizó por las actividades agropecuarias; servicios financieros, seguros bienes muebles; comercio, restaurantes y hoteles; y transportes, almacenamiento y comunicaciones. En medio de estos dos agrupamientos encontramos zonas metropolitanas y de desarrollo industrial más dinámicas del país para aquellos años: el Estado de M éxico y el Distrito Federal en el centro del país, así como Nuevo León en el nortedel país. En tanto que entidades como Chiapas y Guerrero, se caracterizaron por las actividades agropecuarias las cuales reflejaban una estructura poco presente en otros sectores.

Para el año 1995, la clasificación integró dos grupos principales (ver figura 12): la clase 1 que concentró a las entidades de: Aguascal ientes, Baja California, Coahuila, Chihuahua, Guanajuato, Hidal go, Jalisco, Estado deM éxico, Morelos, Nuevo León, Puebla, Querétaro, San Luis Potosí, Sonora, Tamaulipas, Tlaxcala, Veracruz y Yucatán. En una oposición menos marcada cuando 
comparamos con 1970, encontramos a la clase 3, integrada por los estados de: Baja California Sur, Colima, Chiapas, Durango, Guerrero, Michoacán, N ayarit, Oaxaca, Sinal oa, Tabasco y Zacatecas. Las otras tres clasificaciones son: Ia clase2, únicamentecon el Distrito Federal; la clase 4 con el estado de Quintana Roo y la clase 5 con el estado de Campeche.

Por otro lado, Ios sectores característicos para este año de 1995 no muestran el contraste de 25 años atrás. En la clase 1 el sector característico fue la industria manufacturera con un promedio en los cocientes de localización para este sector de 1.18 contra .84, quefueel promedio general, y en la clase 4 el sector característico fue el agropecuario, cuyo promedio en términos del cociente de localización fue de 2.32, contra el promedio general, de 1.49 .

El gran cambio a lo largo del periodo estudiado fue la emergencia de un sector manufacturero presente prácticamenteen todo el país. Así en 1995, podemos ver que se presentaron estructuras regionales con fuerte presencia en las actividades agropecuarias, y por el otro lado, el sector de las manufacturas se instaló en casi dos terceras partes del total de las entidades del país, podemos decir queel país seindustrializó en la medida en quela apertura se fuegeneralizando y los movimientos decapital fueron aumentando, como lo confirma el incremento delas inversiones extranjeras directas en la última etapa del periodo estudiado (secretaría de Economía, DGIE, 2002).

\section{LA DISTRIBUCIÓN TERRITORIAL Y SUS TENDENCIAS}

Para concluir el análisis en la especialización regional de la producción para el periodo de 1970 a 1995, es importante situar los cambios en un nivel territorial y tratar de especular sobre las tendencias futuras de la organización territorial de la producción.

El año 1970 parece distante para evaluar la nueva organización territorial del país, sin embargo, ese fue el inicio de una década quesecaracterizó por los cambios y las crisis dela economía mundial, y en particular en México. Los cambios generales fueron el inicio de un proceso de internacionalización de las economías 
nacionales; el comienzo del desarrollo espectacular en las formas de comunicación y la utilización cada vez más acelerada de la telemática en las relaciones económicas. En tanto queen el ámbito nacional fue un periodo en el que el nivel delas reservas petroleras del país contribuyó a profundizar una economía cerrada y mantener una posición independiente de los cambios que experimentaba la economía mundial en aquel momento.

La situación tan particular de la economía mexicana y su equilibrio con el exterior, hacen que 1970 sea un año apropiado para testimoniar las características de los cambios que se pudieron identificar en la estructura de producción existente en 1995. El resultado de este análisis muestra diferencias profundas en la especialización regional de la producción en el país entreambos años, y permite identificar las estructuras de especialización regional transitorias de 1980 y 1985 (no detalladas en este escrito). Si bien estos cambios variaron de intensidad según la dinámica nacional en los diferentes sectores de la producción, las interrelaciones entre los nuevos imperativos de las actividades y las características de las regiones modelan una nueva geografía de la producción en el país.

Con base en lo expuesto en el párrafo anterior, se pueden establecer grandes diferencias entre 1970 y 1995. Con el auxilio delas figuras 9 y 12 es posible identificar cómo en 1970 el país seencontraba dividido en dos clases que territorialmente muestran dos grandes franjas: la primera clase, que integra la península de Baja California y la mayor parte de los estados fronterizos del país y con un nodo importante en el occidente formado por los estados de Jalisco, Colima y Aguascalientes, al que se agregan Morelos y Yucatán. La segunda clase va del océano pacífico desde el estado de Sonora hasta Quintana Roo. Asimismo hay tres estados que presentan diferencias notables en relación con los anteriores agrupamientos; dichas entidades son el Distrito Federal, el Estado de México y Nuevo León. Los otros dos estados atípicos con respecto a la especialización del producto interno bruto nacional son Chiapas y Guerrero. 
Por otro lado, el grupo de entidades en expansión que se ubica predominantementeen la clase 1, tiene como característica principal una al ta especialización en la generación de producto en los sectores construcción, comercio, restaurantes y hoteles, y transportes y almacenamiento. El grupo de entidades que liga el noroeste con el sudeste del país tiene como característica una alta especialización en la generación de productos agropecuarios y en la prestación deservicios financieros, seguros y bienes inmuebles. Estos estados tuvieron una participación baja en el comercio, restaurantes y hoteles, y transportes y almacenamiento. La zona metropolitana del valle de México, que constituye la clase 3 se caracteriza por una participación al ta en las actividades de servicios comunales, sociales, y personales y en manufacturas. Los estados restantesson: Nuevo León, quetieneuna composición cercana al valle de México, y además Chiapas y Guerrero. En suma hay, una especialización manufacturera quese concentra en el Distrito Federal, Estado de México y Nuevo León; un segundo grupo de entidades en expansión formado por las entidades en crecimiento, en donde destacan las de la frontera norte y la importante urbanización que experimentó el occidente durante el periodo previo. El tercer grupo lo integran en su mayoría las entidades de especialización ligadas predominantemente a las actividades pecuarias, en las queseencuentran las deuna importanteagricultura comercial en el noroeste hasta entidades con prácticas más tradicionales. El último grupo son los estados de Chiapas y Guerrero, con un perfil más difuso en lo que toca a las actividades y su composición.

En 1995el país pasó por una crisis de confianza muy importante y entró, debido a las medidas de ajuste estructural emprendidas después de 1984, a una etapa de reestructuración quesereflejó de manera diferente en cada una de las regiones del país.

La figura 12 permiteobservar la regularidad en la distribución de las clases 1 y 3 en 1995, en contraposición a lo sucedido en 1970; las diferencias entre estas dos clases que integraron a 29 de los 32 estados del país, fueron menores. Mientras que los estados atípicos del conjunto fueron: Campeche que se relaciona con el 
petróleo; Quintana Roo, definitivamenteespecial izado en el turismo; y el Distrito Federal convertido para esteaño en una clasemedia entre los dos grandes conjuntos que fueron las clases 1 y 3 . Entre estas dosúltimas clases la diferencia importante seestablecea partir de la especial ización en las manufacturas que tiene la clase uno y la especialización en la producción agropecuaria quetienela clase 3.

\section{Conclusiones}

Muchas reflexiones se podrían obtener de los resultados expuestos, pero se pueden resumir en dos grandes conclusiones: La economía mexicana setransformó estructuralmenteal aumentar la participación en el sector manufacturero duranteel periodo tratado en este escrito. Esta transformación tuvo resultados diferentes, formas características propias decada una delas regiones del país; sin embargo, la industria mostró un despliegue importante que logró marcar cambios en la especialización regional del PIB para 1995. Habría que investigar si la estructura de este año reflejó la inestabilidad macroeconómica o si las estructuras sectorial es aún con baja actividad marcaron sus perfiles correctamente para dicho año.

La segunda conclusión es que la economía mexicana se logró diversificar y densificar en el territorio nacional. Es evidente que el resto de los sectores, como son el de comercio y de servicios, han logrado homogeneizar su presencia en el territorio nacional. En estesentido, el tipo decomercio, el tipo deservicio y el uso de losmedios decomunicación y telecomunicación modernos podrían ser el origen de la nueva división que va a caracterizar a la geografía de la producción después de 1995.

\section{BiBLIOgRAFÍA}

Aguilar, A.G. y Graizbord B. 1996. Las ciudades intermedias y el desarrollo regional en M éxico", Conaculta-UNAM-COLMEX, México. 
Azúa, J. 2000. A lianza competitiva para la nueva economía, M cGrawHill, Madrid.

Cárdenas, E. 1996. La política económica de M éxico, 1950-1994, FCECOLMEX, México.

Cervantes, G. 1996 “Cambio estructural en el sector externo de la economía mexicana”, Comercio Exterior, vol. 46, núm. 3, pp. 175-186.

Fischer, A., 1994. Industrie et espace geographique, Masson-Geographie, París, Francia.

Hiernaux, D. 1998. "Reestructuración económica y cambios territoriales en M éxico. Un balance 1982-1995", en De Mattos C., D. Hiernaux, D. Restrepo. Globalización y territorio, Impactos y perspectivas , FCE-Universidad Católica de Chile, Santiago, 92-119.

ITESM. 1999. La competitividad de los estados mexicanos , Monterrey. Lebart, L., A. Morineau y M. Piron. 1995. Statistique exploratoire multidimensionelle, Dunod, París.

Lora, E. y E. Barrera. 1998. “El crecimiento económico en A mérica Latina después de una década de reformas estructurales", en BID, A mérica Latina después delas R eformas, Pensamiento I be roamericano, número especial, pp. 55-86.

Lustig, N. 1994. Hacia la reconstrucción de una economía, FCECOLMEX, México.

Rendón, A. 1997. “Grupos económi cos en la década de los ochenta. Estrategias de diversificación o especialización", E conomía Teoría y Practica, núm. 8, pp. 53-70.

Ruiz Durán, C. 1999. Territorialidad, industrialización y competitividad local en el mundo global, en Dinámica regional y competitividad industrial, UNAM - Fundación Friederich EbertEd. Jus, México, pp. 13-56.

Secretaría de Economía y DGIE. 2002. Informes de la inversión extranjera por entidad federativa, México. 